\title{
Experimental analysis on air-to-water sound transmission loss in shallow water
}

\author{
Zhaohui Peng*, Lingshan Zhang, and Guangxu Wang \\ State Key Laboratory of Acoustics, Institute of Acoustics, CAS, No. 21 North 4th Ring West Road, 100190 Beijing, China
}

\begin{abstract}
Research on the propagation characteristics of air-to-water sound transmission is of great importance to the detection of aerial target from underwater. In order to study the propagation characteristics of air-to-water sound transmission in shallow water, an experiment was conducted in the South China Sea in March, 2013. During the experiment, signals transmitted by a hooter hung on a research ship were received by an underwater hydrophone. The source signals were also recorded by a microphone under the hooter. The distance between the hooter and the hydrophone was from $2.4 \mathrm{~km}$ to $9.8 \mathrm{~km}$ approximately. Data measured during the experiment is analysed in this paper. The transmitted signals and received signals were carefully filtered and calibrated one-by-one. It is shown that the SNRs at $9.8 \mathrm{~km}$ are up to $12 \mathrm{~dB}$ and $18 \mathrm{~dB}$ at the frequencies $128 \mathrm{~Hz}$ and $256 \mathrm{~Hz}$, respectively. The experimental air-to-water sound transmission loss is estimated up to $9.8 \mathrm{~km}$ in range, which has clear oscillation structure. A wavenumber integration approach is used to predict the air-to-water transmission losses, which are in good agreement with experimental data.
\end{abstract}

\section{Introduction}

Because the ratio of acoustic characteristic impedances of air and water is about $1 / 3600$, only little energy generated by an airborne source can transmit into water. Usually, the signal-to-noise ratio of the sound signals received by the underwater sensors is very low, which makes it very difficult to do further research on the airto-water sound propagation. However, with rapid development in the aircraft industry, research on the underwater sound field excited by airborne source has been paid much attention.

Transmission of sound into water from airborne sources has been studied since 1950s. The ray theory, the normal-mode theory, and the wavenumber integration approach have been developed in the theoretical research. Hudimac [1] studied the sound intensity in the water due to a point airborne source by the ray theory. Urick [2] demonstrated that there are four ways for sound excited by an airborne source to reach an underwater hydrophone: via a direct refracted path, via one or more bottom reflections, via the so-called lateral wave, and via sea scattering. For shallow water with a depth-varying sound-speed profile above a stratified seabed, Chapman [3-4] presented the normal-mode theory of the transmission of sound from an airborne source into an ocean, and he then developed an air-to-water transmission program which replaces the source in the air with an equivalent source in the water. Yan [5-6] simulated the air-to-water sound transmission in shallow water by the BDRM model and also studied the effect of a randomly rough boundary on the sound field using the coupled normal-mode method. Based on the wavenumber integration approach, Schmit [7] developed a computer program referred to as OASES, which can be used to predict the air-to-water transmission. Zhang and Peng [8-9] used a self-consistent perturbation approach which combined the wavenumber integration approach and the perturbation method to study the air-to-water sound transmission with a rough sea surface, and they also analysed the effect of the height of the source on air-to-water transmission.

On the experimental research, Urick [2] concluded that ray contours gave a fair fit to the total field in the sea excited by an aircraft in the air. Lubard [10] carried out an experiment where the distance between the source and receiver was $48 \mathrm{~m}$ with a loudspeaker as the airborne source and he also took the ocean roughness into account. Buckingham [11] investigated the properties of the sound field in a viscous medium excited by a moving source in the air, and introduced an asymmetry into the attenuation coefficient of propagating waves. Peng [12] conducted an experiment using a high-power loudspeaker as an airborne source and a horizontal line array (HLA) lay on the sea bottom near the source. In that experiment, the estimated locations of the airborne source was in agreement with the GPS measurements. In the above experiments, the receiver was located near the source within $1 \mathrm{~km}$. In another experiment conducted by Wang [13] during which linear frequency modulation (LFM) signals were transmitted and an HLA was deployed, the distance between the loudspeaker and the HLA was up to $4 \mathrm{~km}$. The transmission loss calculated by a wavenumber integration model was in good

Corresponding author:pzh@mail.ioa.ac.cn 
agreement with the experimental value. Since the underwater field excited by linear frequency modulation (LFM) signals, both experimental and theoretical data were averaged over a certain bandwidth. Hence the transmission loss curves showed a relatively smooth change with the distance.

In order to perform further analysis on the fine structure of air-to-water sound transmission in shallow water, the research on the underwater field excited by single-frequency signals is necessary. Therefore, we conducted an air-to-water experiment. A hooter hung on the research ship was used as the airborne source which transmitted multi-frequency signals, and a microphone under the hooter was used to record the direct wave signals. A hydrophone laid on the sea bottom was used to receive signals, and the distance between the hooter and the hydrophone was up to $9.8 \mathrm{~km}$. Experimental airto-water sound transmission loss is obtained by analysing data from this experiment in this work. First, we extract line spectra features of power spectrum density of transmitted and received signals, and set up parameters of narrow-band filters which are used to process signals based on line spectra features. Second, the total powers of processed transmitted and received signals are calculated as sound pressure levels. And then calibrated signals recorded by the microphone are used to obtain experimental air-to-water sound transmission losses at $128 \mathrm{~Hz}$ and $256 \mathrm{~Hz}$. Finally, we compare experimental air-to-water sound transmission losses with theoretical ones, and give a physical explanation for transmission characteristics of the underwater field excited by an airborne source.

The environment in this paper is assumed to be range-independent, that is, the properties of air, water and the sea bottom are all depth-dependent. Therefore, the wavenumber integration approach [14] is applicable to predict the air-to-water sound transmission loss. Schmidt [7] developed a general purpose computer code referred to as OASES based on the wavenumber integration approach, which can accommodate propagation within solid, fluid, and air layers. In this paper, the theoretical air-to-water sound transmission loss is calculated by OAST, one module of OASES, which is able to model the air-to-water sound transmission in shallow water.

\section{Introduction of the experiment}

In March 2013, an experiment was conducted in the South China Sea. The sea was calm. The seabed of the experiment area is relatively flat and the average water depth is $93.4 \mathrm{~m}$. A hooter with height $1.1 \mathrm{~m}$ was hung $3.0 \mathrm{~m}$ above the sea surface used as source. A microphone was placed $0.4 \mathrm{~m}$ below the hooter to receive the direct wave signals. A hydrophone was laid on the sea bottom to receive the transmitted signals. Figure 1 shows a schematic of the experimental layout. The position between the research ship and the hydrophone is shown in Fig.2, where the hydrophone is located at the origin, and the solid curve represents the trajectory of the research ship. During the experiment, the research ship went outward from $2.4 \mathrm{~km}$ to $9.8 \mathrm{~km}$ in range.

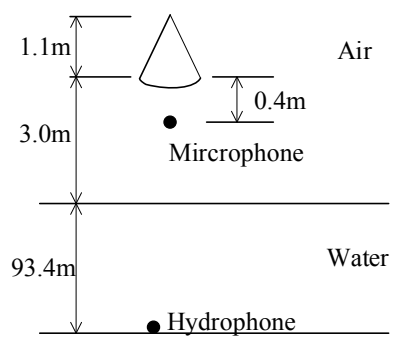

Fig. 1. Schematic of the experimental layout.

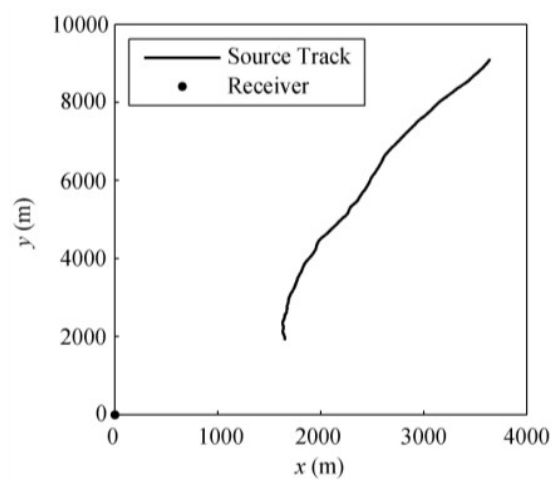

Fig. 2. Location of the hydrophone and the trajectory of the research ship.

During the experiment, total 349 signals were transmitted. Each signal, consisted of $1 \mathrm{~s}$ pulse, $2 \mathrm{~s}$ suspension, $5 \mathrm{~s}$ pulse, $2 \mathrm{~s}$ suspension, and $1 \mathrm{~s}$ pulse, was transmitted every minute. The signal recorded by the microphone is shown in Fig. 3 and the power spectrum density of the middle $5 \mathrm{~s}$ pulse was shown in Fig. 4 . It is shown that the energy of the transmitted signal concentrates at $128 \mathrm{~Hz}$ and its multiples. The source levels of the signals were then analyzed [15].

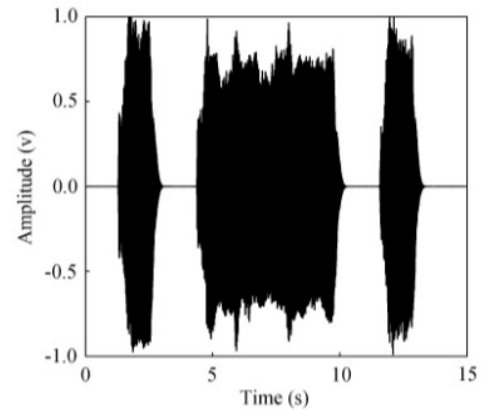

Fig. 3. Signals transmitted by the hooter and recorded by a microphone below the hooter.

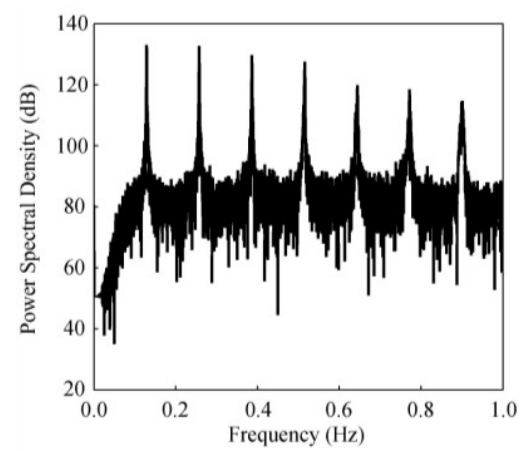

Fig. 4. The power spectrum density of the middle $5 \mathrm{~s}$ pluse. 


\section{Analysis of transmission loss}

Transmission loss $[15,18]$ is a quantitative measure of the reduction in sound intensity between two points, normally the sound source and a distant receiver. In the case of air-to-water transmission, because the acoustic impedance of air is different from that of water, and the reference pressures for calculating the sound pressure level in these two media are different, it is incorrect to simply subtract the sound pressure level at the receiver in the water from that produced at a distance of $1 \mathrm{~m}$ from source in the air.

The transmission loss is defined as

$$
T L=10 \log _{10}\left(\frac{I_{1}}{I_{r}}\right)
$$

where $T L$ is the transmission loss, $I_{1}$ is the acoustic intensity produced at a distance of $1 \mathrm{~m}$ from source in the air, and $I_{r}$ is the acoustic intensity at point $r$. In an infinite, homogeneous medium, for a plane wave, the relationship between the acoustic intensity $I$ and the sound pressure $p$, the density of the medium $\rho$ and the sound speed $c$ can be written as

$$
I=\frac{p^{2}}{2 \rho c}
$$

Substituting Eq. (2) to Eq. (1), the air-to-water sound transmission loss can be rewritten as,

$$
T L=10 \log _{10} p_{\mathrm{a}}^{2}-10 \log _{10} p_{\mathrm{w}}^{2}+10 \log _{10} \frac{\rho_{\mathrm{w}} c_{\mathrm{w}}}{\rho_{\mathrm{a}} c_{\mathrm{a}}}
$$

where subscripts a and $\mathrm{w}$ indicate acoustic properties in the air and water, respectively. As the reference pressures in two media are different, which are $p_{\text {aref }}=20 \mu \mathrm{Pa}$ in the air but $p_{\text {wref }}=1 \mu \mathrm{Pa}$ in the water, sound pressure level $(S P L)$ is used [16]. Thus, Eq. (3) can be written as,

$$
T L=S P L_{\mathrm{a}}-S P L_{\mathrm{w}}+10 \log _{10} \frac{\rho_{\mathrm{w}} c_{\mathrm{w}}}{\rho_{\mathrm{a}} c_{\mathrm{a}}}+20 \log _{10} \frac{p_{\text {aref }}}{p_{\text {wref }}}
$$

where the $S P L_{\mathrm{a}}$ and $S P L_{\mathrm{w}}$ are the sound pressure level of the transmitted and received signals, respectively.

\subsection{Frequency analysis of transmitted and received signals}

The middle $5 \mathrm{~s}$ pulses were analyzed in this paper. First, power spectrum density (PSD) of transmitted and received signals at $128 \mathrm{~Hz}$ and $256 \mathrm{~Hz}$ are calculated, as shown in Fig.5 and Fig.6, respectively. It is evident that the power spectrum density has a peak value at $128 \mathrm{~Hz}$ or $256 \mathrm{~Hz}$ for every signal, and the frequency and width of each peak are different for each signal. The main reason is that during the experiment, the aeration quantity of the hooter changes, the air pressure is different when the hooter transmits different signals.
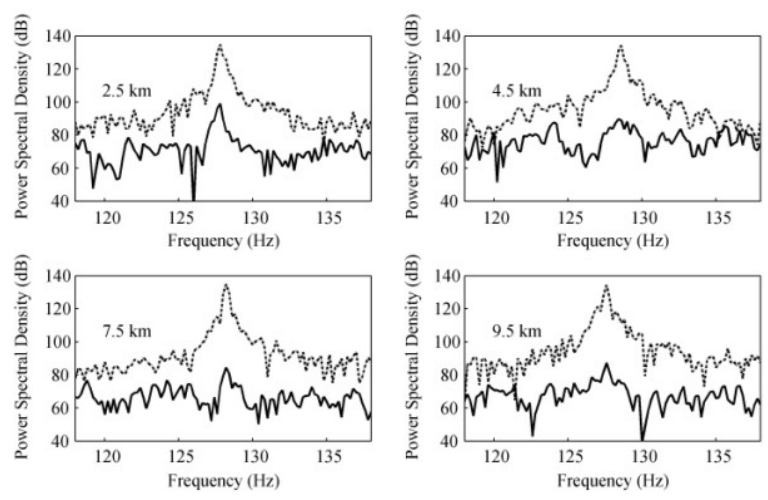

Fig. 5. PSD of transmitted and received signals at $128 \mathrm{~Hz}$.
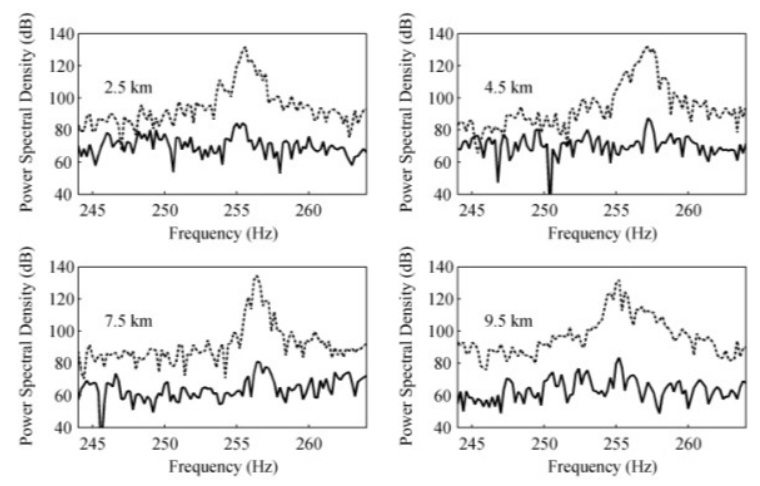

Fig. 6. PSD of transmitted and received signals at $256 \mathrm{~Hz}$.

The narrow-band filters based on the characteristics of each signal's power spectrum density are used to calculate the sound pressure level and avoid interference of environmental noise. A transmitted signal and its corresponding received signal are processed by the same filter.

\subsection{Analysis of source level}

The sound pressure levels ( $\left.S P L_{\mathrm{a}}\right)$ of the each transmitted signal at $128 \mathrm{~Hz}$ and $256 \mathrm{~Hz}$ are calculated and shown in Fig.7 and Fig.8, where the horizontal axis is the distance between the source and the underwater hydrophone. It is clearly shown that the $S P L_{\mathrm{a}}$ exhibits fluctuation with range and the fluctuation at $256 \mathrm{~Hz}$ is rapider than that at $128 \mathrm{~Hz}$.

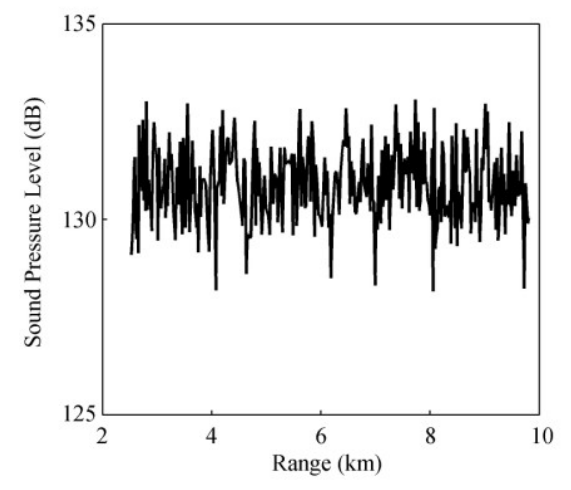

Fig. 7. $S P L_{\mathrm{a}}$ of transmitted signals at $128 \mathrm{~Hz}$. 


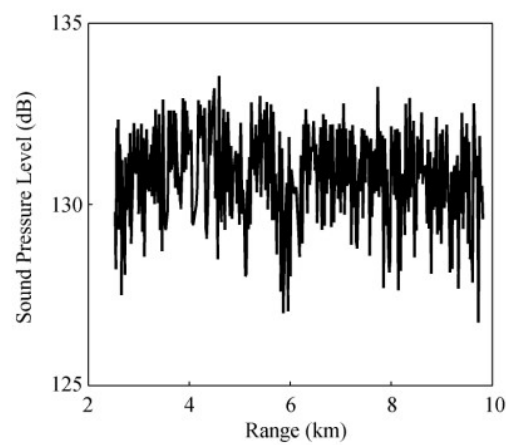

Fig. 8. $S P L_{\mathrm{a}}$ of transmitted signals at $256 \mathrm{~Hz}$.

\subsection{Sound pressure level of received signals}

Using the same narrow-band filters as those used to process transmitted signals, The sound pressure levels $\left(S P L_{\mathrm{w}}\right)$ of received signals were calculated and shown in Fig.9 and Fig. 10 at $128 \mathrm{~Hz}$ and $256 \mathrm{~Hz}$, respectively. It is clearly shown that $S P L_{\mathrm{w}}$ at these two frequencies fluctuates with range, and it oscillates more rapidly at $256 \mathrm{~Hz}$ than at $128 \mathrm{~Hz}$.

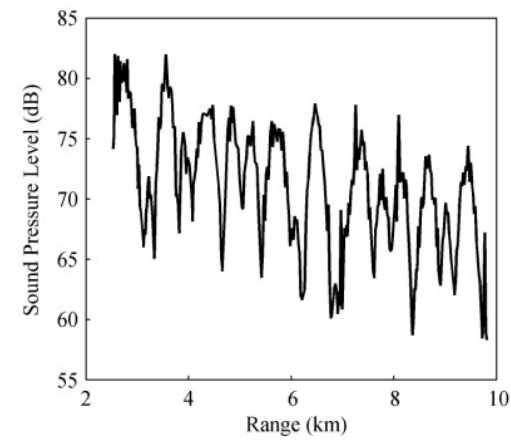

Fig. 9. $S P L_{\mathrm{w}}$ of received signals at $128 \mathrm{~Hz}$.

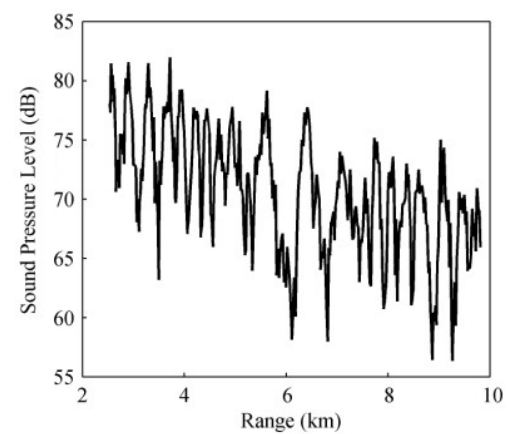

Fig. 10. $S P L_{\mathrm{w}}$ of received signals at $256 \mathrm{~Hz}$.

\subsection{Air-to-water sound transmission loss}

In the above analysis, sound pressure levels for sources in the air and receivers in the water at $128 \mathrm{~Hz}$ and 256 $\mathrm{Hz}$ have been obtained. Then, the air-to-water sound transmission loss can be computed by Eq. (4).

The theoretical air-to-water sound transmission loss is calculated by OAST. The environment is schematically shown in Fig.11, and the sound speed profile measurement is given in Fig. 12.

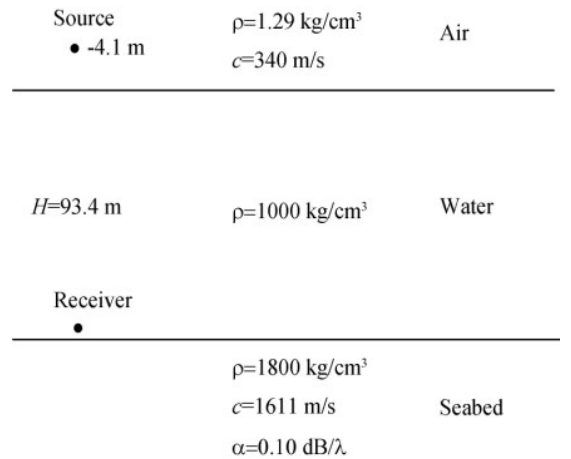

Fig. 11. Environment model parameters for OAST.

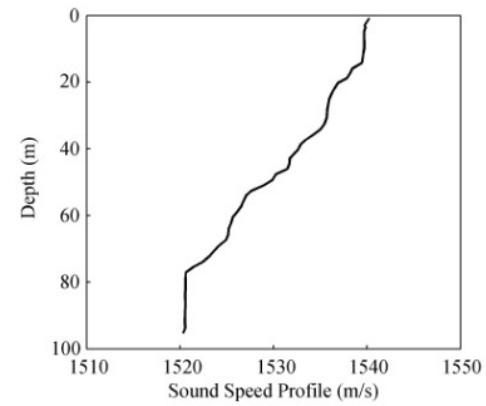

Fig. 12. Measured sound speed profile during the experiment.

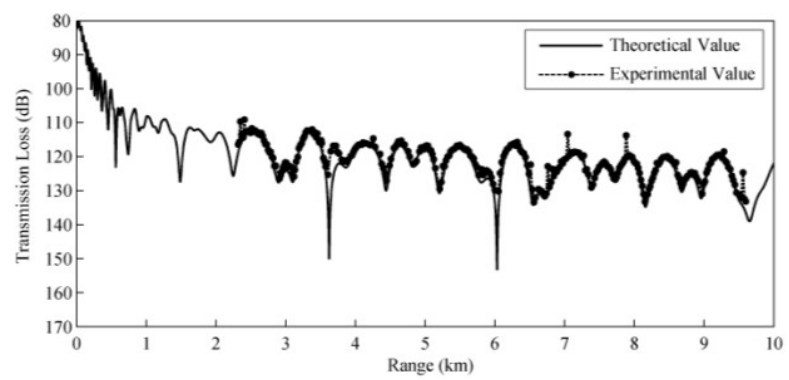

Fig. 13. Modal/Data Comparison of air-to-water sound transmission loss at $128 \mathrm{~Hz}$.

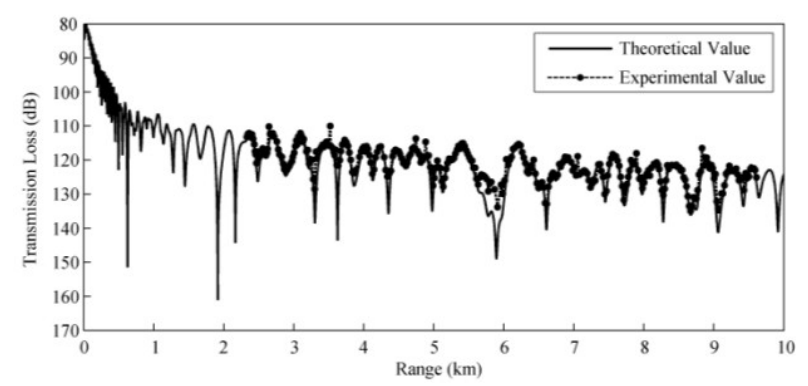

Fig. 14. Modal/Data Comparison of air-to-water sound transmission loss at $256 \mathrm{~Hz}$.

Both experimental and theoretical air-to-water sound transmission losses are presented in Fig.15 and Fig.16 at frequencies $128 \mathrm{~Hz}$ and $256 \mathrm{~Hz}$, respectively. In these two figures, the horizontal axis is the distance between the source and the underwater hydrophone, with the dotted curves corresponding to the experimental results and the solid curves corresponding to the theoretical results. It is clear that the experimental and theoretical results are in agreement at both frequencies $128 \mathrm{~Hz}$ and $256 \mathrm{~Hz}$ with consistent oscillation structures along the range. 


\section{Conclusions}

In this paper, the experimental data of the air-to-water experiment conducted in the South China Sea, March 2013, is analysed to study the characteristics of the airto-water sound propagation at frequencies $128 \mathrm{~Hz}$ and $256 \mathrm{~Hz}$, with the relative distance between the source and the underwater hydrophone up to $9.8 \mathrm{~km}$. Power spectrum densities of transmitted and received signals are analyzed for different distances between the research ship and the underwater hydrophone. For remote received signal, $9.5 \mathrm{~km}$, its power spectrum density indicates that the SNR is up to $12 \mathrm{~dB}$ and $18 \mathrm{~dB}$. Meanwhile, it is evident that the frequency of the peak value and the width of the peak are different for signals transmitted at different ranges, so the analysis and compensation to the amplitude and frequency of transmitted signal are done for every signal to obtain higher quality air-to-water sound transmission losses. The wavenumber integration approach (OAST) is used to calculate the theoretical air-to-water sound transmission loss, and it gives a physical explanation for characteristics of the air-to-water sound propagation. Good agreement between the experimental and theoretical air-to-water transmission loss is observed.

\section{Reference}

1. Hudimac A. A., J. Acoust. Soc. Am., 29, 8 (1957)

2. Urick R. J., J. Acoust. Soc. Am., 52, 3 (1972)

3. Chapman D. M. F. and Ward P. D., J. Acoust. Soc. Am., 87, 2 (1990)

4. Chapman D. M. F., Thomson D. J and Ellis D. D., J. Acoust. Soc. Am., 91, 4 (1992)

5. J. Yan and Z. Peng, Tech Acoust (in Chinese), 21, (2002)

6. J. Yan and R. Zhang, Pro. Nat. Sci. (in Chinese), 13, 3 (2003)

7. Schmidt H., Oases version 3.1 user guide and reference manual, (2005)

8. L. Zhang and Z. Peng, SICIENTIA SINICA Physica, Mechanica \& Astronomica (in Chinese), 44, 9 (2014)

9. L. Zhang and Z. Peng, Chin. Phys. Lett., 31, 9 (2014)

10. Lubard S. C. and Hurdle P. M., J. Acoust. Soc. Am., 60, 5 (1976)

11. Buckingham M. J., J.Acoust. Soc. Am., 114, 6 (2003)

12. Z. Peng, Z. Li, and G. Wang, Chin. Phys. Lett., 27, $11(2010)$

13. G. Wang, Z. Peng, and R. Zhang, Acta Acoustic (in Chinese), 36, (2011)

14. Jensen F. B., Porter M. B., and Kuperman W. A., et al, Computational ocean acoustics, (New York: Springer Science Business Media, 2011)

15. G. Wang, Phd Dissertation (in Chinese), (Graduate University of Chinese Academy of Sciences, 2010)
16. B. Liu and J. Lei, Principle of acoustics in water (in Chinese), (Harbin: Harbin Engineering University, 2009)

17. Y. Ding, X. Gao. Digital signal processing (in Chinese), (Xi'an: Xi Dian University, 2003) 